\title{
LA GESTIÓN PRIVADA DEL GOBIERNO DE INTERNET: LA CAPACIDAD DE AUTODETERMINACIÓN EN JUEGO
}

\author{
The private ordering of Internet governance: \\ powers of self-determination at stake
}

\author{
PATRICIA MINDUS \\ Universidad de Uppsala
}

Cómo citar/Citation

Mindus, P. (2016). La gestión privada del gobierno de Internet: la capacidad de autodeterminacón en juego. Revista de Estudios Políticos, 173, 113-140. doi: http://dx.doi.org/10.18042/cepc/rep.173.03

\section{Resumen}

Para entender mejor qué problemas plantea a día de hoy la tecnología en relación a las libertades políticas, entendidas como autodeterminación, la atención debe desplazarse desde el nivel de los contenidos de Internet hacia los elementos básicos que definen su arquitectura y gobernanza (CIR, protocolos, relaciones de poder, etc.). La ciencia política convencional ha ignorado durante mucho tiempo el campo del gobierno de Internet. A pesar de ello, existe un interesante debate sobre cómo se debe gestionar este nuevo mundo, constituido por la infoesfera, que tenemos en común. El artículo muestra por qué el debate sobre el control de Internet se plantea frecuentemente de manera errónea: al prestar demasiada atención a los actores institucionales tradicionales se ha obviado que las «sedes de poder» ahora se encuentran más repartidas. Para poder moverse a través del modelo de gestión privada que actualmente caracteriza Internet de una manera respetuosa con los derechos fundamentales hace falta repensar las relaciones entre los poderes sociales.

\section{Palabras clave} cracia.

Libertad política; gobierno de Internet; CIR; arquitectura de Internet; demo- 


\begin{abstract}
To better understand what issues today's technologies raise in relation to political liberty, understood as self-determination, attention should shift from the level of contents of the Internet to its more basic architecture and governance (critical internet resources [CIR], protocols, governance ecology, etc.). Mainstream political science has for a long time eschewed the field of Internet governance. There is nonetheless an interesting debate on how we should handle this new world we have in common that is constituted by the infosphere. The paper shows why the debate on the control of the Internet is often misleading: too much attention on traditional institutional actors has obscured the fact that the «seats of power" are now elsewhere. In order to move within the private ordering that currently characterizes Internet governance, in a way that is respectful of fundamental rights, we need to rethink the relationships between social powers.
\end{abstract}

\title{
Keywords
}

Political freedom; Internet Governance; CIR; Internet architecture; Democracy. 


\section{SUMARIO}

I. UN NUEVO ÁMBITO DE LA POLÍTICA. II. CONTROLAR LA ESTRUCTURA. III. MULTISTAKEHOLDERISM Y CENTROS DE PODER. IV. PODER DE Y PODER SOBRE. BIBLIOGRAFÍA.

\section{UN NUEVO ÁMBITO DE LA POLÍTICA}

La infoesfera es ese nuevo mundo que habitamos conjuntamente con otros inforgs, ya sean agentes naturales o artificiales. En los bits se mezclan el fuero interno, tradicionalmente influenciado por el poder ideológico a través de la dirección de las almas, las mentes, los pensamientos y los valores, y el fuero externo, normalmente bajo el control del poder económico y político que domina los cuerpos, las necesidades y las actividades materiales. El advenimiento de la Internet de las cosas y de la robótica civil de masas hace esto evidente al hacer desaparecer la propia distinción entre lo on- y lo off-line. Gestionar un entorno así es, sin lugar a dudas, una tarea complicada.

Mientras que, inicialmente, la red se consideraba un reino habitado por pioneros librepensadores que habían creado colectivamente reglas y normas para ordenar este nuevo paisaje, a partir de ahora las luchas que se desarrollen en Internet estarán protagonizadas por jugadores más importantes, in primis los gobiernos y las grandes fortunas [...]. En el centro de todo está la cuestión de quién puede intervenir, y de qué manera, y quién tiene acceso a qué información [...]. La red es, al mismo tiempo, liberadora y feudal ${ }^{1}$.

La cuestión que se plantea es, por tanto: ¿̨liberadora para quién, y respecto de qué?

Como medio de comunicación que es, Internet permite a sus usuarios, de diversas formas, gozar de "poder para» expresarse, asociarse, calcular, etc., pero en determinadas circunstancias, esa capacidad de algunos puede implicar también el ejercicio de un "poder sobre» los demás. Como sostiene uno de los miembros del Working Group on Internet Governance (WGIG), Don MacLean, «además de dar la oportunidad a los usuarios individuales y a los

1 Faris y Heacock (2013: 3). 
grupos de crear contenidos, desarrollar servicios y ejercitar libertades fundamentales, Internet también otorga la posibilidad de monopolizar los mercados, controlar el acceso a la información y negar derechos humanos esenciales» ${ }^{2}$. El reto que plantean las nuevas tecnologías en relación a las libertades políticas es cómo regular un ámbito así. Si la libertad política, o la autonomía colectiva, presupone que «todo ser humano debe participar de manera directa o indirecta en la conformación de las normas que, a continuación, regularán su conducta en la esfera que no está reservada a la competencia exclusiva de su jurisdicción individual ${ }^{3}$, nos encontramos de frente con una cuestión clave para la democracia.

Las características de Internet - la maleabilidad de su contenido y su arquitectura difusa - pueden fácilmente llevar a pensar, de manera equivocada, que «nadie controla la red» aunque existan mecanismos de coordinación, en algunas ocasiones centralizados, sobre diversas áreas técnicas y administrativas necesarias para mantener Internet funcionando. «Es ingenuo suponer que la red es como es, o que permanecerá fiel a los mismos principios de hoy, por razones de necesidad técnica ${ }^{4}$. Más aún, en los últimos veinte años Internet ha cambiado sustancialmente, y con ella los hábitos de quienes la usan, lo cual ha modificado la manera en la que es gestionada: hay quien habla de additional levers of control que han venido creándose mediante algoritmos automatizados para regular el contenido online 5 . Estas circunstancias han generado nuevas estructuras de poder: "el nexo entre las comunidades profesionales, los puntos de control técnico y la regulación privada ha sido articulado dentro de un [...] modelo de ordenación del contenido de la red donde una pequeña élite profesional controla los límites del global default de la expresión libre» ${ }^{6}$.

Para entender mejor los problemas que las nuevas tecnologías suponen para la libertad política, entendida como autodeterminación, conviene fijarse en un ámbito específico: la gestión de la red (Internet governance). Para empezar a entender en qué términos se plantea la relación entre la libertad política y las nuevas tecnologías, lo primero es alejarse del mito de la neutralidad de la tecnología ${ }^{7}$. Como ha escrito la especialista en Internet governance, Laura DeNardis, al igual que sucede con cualquier otro instrumento técnico, los de Internet —incluso aquellos que son indiferentes al contenido y, por tanto, de

\footnotetext{
MacLean (2005: 31).

Bobbio (2009: 321).

Wagner (2013: 201).

DeNardis (2012).

Wagner (2013: 14).

7 Kallinikos (2010: 3). «Los aparatos como medios nunca son neutrales. Hacen posibles algunas cosas, y evitan la realización de otras». Para una explicación más detallada me permito remitirme a Mindus (2014 y 2015).
} 
carácter meramente formal o estructural — no son «instrumentos aislados ni neutrales, sino que reflejan las opciones de control escogidas por sus diseñadores», por lo que «esta estructura técnica no es ajena a la política ni a la cultura, sino que se basa en valores y preferencias políticas que, en última instancia, estructuran la libertad personal en la red y el desarrollo de esta [...]: las riendas de la governance también manejan la estructura técnica de Internet» ${ }^{8}$.

Aunque es algo fundamental para el funcionamiento de la red, tal y como la entendemos hoy, la Internet governance se desarrolla generalmente fuera de la visibilidad pública. Esto no sirve sino para fomentar la idea de que, cualquiera que sea la naturaleza de la governance en cuestión, esta no terminará de ajustarse a la definición de la teoría democrática del siglo xx del «gobierno público en público». De hecho, existen varios problemas que aparecen directamente vinculados con las características que hacen de Internet la red de redes. La gestión de la red ${ }^{9}$ se basa en normas, sistemas de reglas y prácticas que, en su nivel más alto, se contienen en leyes nacionales e internacionales, ya se trate de acuerdos de cooperación entre Estados para perseguir más allá de las fronteras los crímenes cometidos a través de la red, o de la garantía que permite a cualquiera expresarse a través de estas redes gracias al art. 27 de la Declaración Universal de Derechos del Hombre.

El mismo concepto de Internet governance es objeto de disputas políticas, que a menudo se reducen a la dicotomía, demasiado rígida, entre tecno-deterministas como son los ciberlibertarios o los ciberconservadores ${ }^{10}$. Ya en junio de 2005, el Report of the Working Group on Internet Governance (WGIG) adoptó una definición muy amplia al respecto ${ }^{11}$, que, por otro lado, se planteaba en términos normativos; de hecho, "esta definición refuerza el concepto de inclusividad de los Estados, del sector privado y de la sociedad civil en los mecanismos de gestión de la red. Esta definición reconoce que cada grupo tiene intereses, roles y (grados de) participación diferentes» ${ }^{12}$. Internet gover-

8 DeNardis (2013: 555-556).

9 Uso aquí la fórmula, ya adoptada por el CNR, "gestión de la red» para traducir «Internet governance», que debe distinguirse del concepto de e-governance, es decir, el uso que se hace de las nuevas tecnologías por parte de la Administración pública y en las funciones de gobierno.

10 Mueller (2010). Sobre la ideología de los ciberlibertarios y del Partido Pirata, véase Burkart (2014).

11 «Internet governance significa el desarrollo y la aplicación, por parte de los Estados, el sector privado y la sociedad civil, cada uno en su ámbito, de principios, normas, reglas, procedimientos para tomar decisiones y programas compartidos, que determinan la evolución y el uso de Internet»: Working Group on Internet Governance (2005).

Ibid. 
nance es un término ambiguo en el que se engloba la gestión de muchísimas cuestiones como «la calumnia, la difamación, el fraude, la falsificación, las políticas fiscales, la protección de la propiedad intelectual, la protección de los consumidores, el control de la estabilidad e integridad del funcionamiento del sistema DNS, la protección de la privacidad de la información personal almacenada en los registros del domain name system, o el control sobre las posibles interceptaciones del tráfico»" ${ }^{13}$. Indudablemente, en todos estos temas es necesario tomar decisiones, pero lo delicado es determinar quién tiene el poder de hacerlo, en el sentido de poseer los medios que sirven para hacerlas eficaces; quién tiene la facultad para ello, o sea, la legitimidad; bajo qué condiciones estas decisiones supondrán poder sobre alguien o algo; cuándo la estructura de poder así establecida determina la subordinación de unos a otros; o, por último, de qué tipo son estos poderes. Todos estos problemas son anteriores al concepto de Internet governance: la propia finalidad política de la gestión de la red hace referencia a un conjunto de cuestiones cuya finalidad es lograr un uso eficiente, además de legal, de Internet.

Teniendo presente que "para muchos, el mismo término ya genera rechazo» ${ }^{14}$, lo primero es apuntar la existencia de distintas taxonomías que sirven para ilustrar cómo se gestiona la red ${ }^{15}$ y que casi siempre intentan arrojar luz sobre «la naturaleza de los jugadores ${ }^{16}$ implicados en esta compleja fórmula de proceso decisional. Esto no debería sorprender, puesto que se trata de un caso de global multistakeholder networked governance, consecuencia — se dice- de la «peer-production of Internet governance». Tres son las características que hacen que esta forma de gestionar la red —independientemente de su misma legitimidad, que igualmente se discute ${ }^{17}$ — se distancie de los diseños propios de la mayoría de los procesos decisionales: i) la asimetría entre unas jurisdicciones aún eminentemente nacionales y el carácter no territorial de la infoesfera; ii) el hecho de que, junto con fórmulas más o menos formales de decision-making, «la mayor parte de la gestión de Internet se realiza de manera descentralizada y emergente; procede de la interacción de decenas de miles de operadores e ISPs - y en ocasiones de los propios usuarios_ que están conectados entre sí a través de protocolos» ${ }^{18}$; y iii) el hecho de que no exista más obligación de emplear determinados servi-

\footnotetext{
13 Cerf (2004: 1362).

14 Mueller (2010: 8).

15 Dutton (2007: 63-81).

16 Dutton (2011); Nash (2013: 441-463).

17 Hintz y Milan (2009: 23-38); Hintz (2007); Hunter (2003: 1149-1183); Weinberg (2000: 187-260). Para una recopilación de perspectivas críticas: http://www. icannwatch.org.

18 Mueller 2010: 9.
} 
cios que la que se deriva de la peer-pressure. No se trata aquí de intentar mostrar en detalle el complejo funcionamiento de la gestión de la red, sino simplemente de evidenciar cómo esta gestión constituye un nuevo ámbito de la política y cómo este es objeto de pugna entre distintos poderes sociales.

Con una finalidad expositiva, y siguiendo la distinción ya clásica de Yochai Benkler de la «red a tres niveles»" ${ }^{19}$, podemos adelantar la conclusión de que la mayor parte de los debates sobre Internet y política, tanto en general como dentro de las instituciones creadas para su "gobierno", como pueda ser el órgano consultivo del IGF (Internet Governance Forum), se concentran en el nivel semántico, el del contenido, ignorando de hecho los otros dos; y ello a pesar de que pueden tener una importante incidencia en aquel. Conviene, por lo tanto, partir de algún ejemplo relacionado con los niveles más "profundos" para aclarar cómo el problema de la «libertad política» está relacionado no tanto y no solo con las muy diversas prácticas de "participación digital» que se desarrollan íntegramente en el nivel de los contenidos de Internet, sino con el poder sobre la backbone infrastructure de la red: el nivel físico y lógico de Internet. La opción por este punto de vista deriva del simple hecho de que, en comparación con el nivel de los contenidos, «los investigadores han dedicado mucha menos atención a la infraestructura de la red $»^{20}$.

\section{CONTROLAR LA ESTRUCTURA}

Desde el punto de vista del nivel físico, Internet es un conjunto de redes que son gestionadas por compañías de telecomunicaciones, propietarias de redes de fibra óptica, cables submarinos o conexiones vía satélite. Todas estas

19 Benkler (2000: 561-579). Conforme a esta descripción, Internet es un conjunto de redes formado por estratos: el primero es «físico» y se refiere a las infraestructuras de comunicaciones: cables, torres, enlaces de radio, hotspots, líneas telefónicas; el segundo es técnico y abarca las reglas y los protocolos (Tcp/Ip, IpV4, IpV6); el tercero es el de los contenidos y lenguajes compartidos en la red (el estándar HTML del W3C, pero también el idioma de los sitios web, e incluso el contenido en sentido estricto: audio, texto, vídeo). Junto con esto hay quien añade un cuarto estrato constituido por las leyes (las normas sobre privacidad, copyright, seguridad, etc.). Preferiría no hablar de ello como un cuarto estrato, ya que se trata del ámbito regulatorio en el que los otros tres tienen que operar. Estos niveles se integran entre sí y se interrelacionan de diversos modos, a partir del trabajo que múltiples sujetos privados y públicos realizan para garantizar la eficiencia, la estabilidad y la evolución de Internet. Este esfuerzo conjunto tiene como objetivo la «gestión de la red».

DeNardis (2013: 566). 
redes físicas deben estar conectadas entre sí para que la red funcione, cosa que sucede gracias a puntos de conexión privados o a través de IXPs (Internet Exchange Points) que implican a una gran variedad de actores ${ }^{21}$. Por ejemplo, uno de estos IXPs es el DE-CIX (Deutscher Commercial Internet Exchange) en Frankfurt, que constituye actualmente el principal hub de la red en Europa con un tráfico que alcanza 3,4 terabytes por segundo. El DE-CIX es propiedad de la Eco Association, una de las mayores asociaciones con fines no lucrativos de la industria de Internet, que a su vez desarrolla actividad de lobbying para alrededor de 500 empresas, sobre todo alemanas. Para conectar redes privadas se requiere obviamente poder desarrollar una interconectividad física, pero se necesitan igualmente contratos que estipulen dónde se localizarán los costes, la responsabilidad, etc. Normalmente estos contratos son de dos tipos, peering y transit, de los que únicamente el segundo prevé la compensación económica a cambio de la prestación de un servicio por parte de un backbone provider. Cualquiera podría argumentar que aquí no hay nada nuevo bajo el sol: desde los tiempos del negotium romano se han venido planteando fórmulas destinadas a resolver eventuales controversias. Sin embargo, la realidad es diferente: «los contratos de interconexión son algo nuevo en la medida en que normalmente no es sencillo identificar las normas aplicables, faltan órganos que se encarguen del control y, además, son poco transparentes ${ }^{22}$.

Si bien está claro que la protección de la infraestructura física es crucial para asegurar una red que funcione, es preciso tener en cuenta que también el plano jurídico influye en la seguridad y fortaleza de la red: esto ya se comprobó en un caso de falta de conectividad producto de una controversia que enfrentó a Cogent y a Sprint ${ }^{23}$. En este punto hay que empezar considerando aquellos problemas con los que los juristas están más acostumbrados a trabajar, como puedan ser las cuestiones sobre competencia o antitrust: hay quien se lamenta de la poca competencia existente en el ámbito operativo de los Internet connection points, que deriva en unos costes de tránsito que deben ser pagados a los backbone providers inasumibles para algunos países en vías de desarrollo. Pero también hay dificultades que se derivan de las situaciones de colaboración voluntaria articuladas mediante los contratos de peering, como las que se han puesto de manifiesto con la reciente disputa entre la empresa de telecomunicaciones francesa Orange y el operador americano Cogent ${ }^{24}$. Todo

\footnotetext{
Wagner y Mindus (2015).

DeNardis (2013: 567).

23 Weiser (2009).

24 La primera se quejaba del hecho de que recibía un flujo trece veces mayor del que enviaba, en buena parte debido a que los datos de Megaupload se transmitían a través de Cogent: Weller y Woodcock (2013).
} 
esto, que está relacionado con las relaciones de poder entre los distintos operadores de la infraestructura, puede potencialmente incidir en el nivel de los contenidos, como evidencia el debate sobre la neutralidad de la red ${ }^{25}$.

$\mathrm{El}$ hecho de que el nivel de los contenidos está influido por todo aquello que sucede en los otros niveles es quizá más evidente en el caso de los CIR (Critical Internet Resources ${ }^{26}$ ). El debate sobre el control de los CIR continúa siendo uno de los «temas centrales en el estudio de la gestión de la red ${ }^{27}$, en la medida en que constituye uno de los grandes asuntos de la política de la red, junto con las controversias relativas a la propiedad intelectual, la seguridad y la censura en la red. Los CIR están relacionados con el sistema de direcciones IP, las funciones IANA, el Domain Name System, el sistema root zone, y también el ASN (Autonomous System Numbers). Dentro de los protocolos y las instituciones responsables de su coordinación ${ }^{28}$ — como IEFE, que se ocupa

25 El concepto de «neutralidad de la red» existe desde mucho antes del nacimiento de Internet: se habló de ello por primera vez en la segunda mitad del siglo xIX, cuando en los Estados Unidos se comenzó a regular la utilización del telégrafo para las comunicaciones. El principio que fundamenta la net neutrality es que el todo tráfico en un determinado medio de transmisión debe ser tratado del mismo modo, por lo que el provider no puede limitar el ancho de banda destinado a acceder a un determinado sitio en relación a otro, o el destinado a la utilización de un determinado software respecto de otro.

26 CIR son aquellos recursos que pertenecen exclusivamente a Internet, excluyendo por lo tanto las infraestructuras físicas, los recursos virtuales y aquellos asociados a la asignación y gestión del espectro electromagnético que no se destinen únicamente a Internet. Si bien algunas definiciones de CIR tienden a incluir también a las infraestructuras de telecomunicaciones y el peering (WGIG), lo habitual es emplear el término para referirse a los recursos lógicos, basados en software, típicos de la arquitectura de la red, que necesitan una coordinación centralizada.

27 Weber (2009: 176).

28 Los protocolos también plantean preguntas interesantes desde el punto de vista político. Por un lado, se admitirá que «el marco normativo más impresionante que ha gobernado Internet hasta hoy está constituido por las instituciones de open standard que han permitido a Internet crecer en modo exponencial, como una red de redes» Weiser (2001: 822). Por otro lado, sigue siendo controvertido qué es un open standard: ¿quiénes son los diseñadores? ¿Quién controla a los diseñadores? ¿Son libres de las reglas de la propiedad intelectual? ¿Cuál debería ser el papel de los gobiernos en la implementación de los standard? ¿Podría ser que las presiones de los gobiernos europeos relacionadas, por ejemplo, con la implementación del standard IPv6, que permitirá elevar el número de potenciales direcciones de Internet de los actuales $2^{32}$ del standard IPv4 a $2^{128}$, no sean casuales sino que estén orientadas a acaparar el mayor espacio posible en función de la arraigada lógica colonial? Véase DeNardis (2011). 
de los protocolos TCP/IP - pero también organismos como W3C, ITU, IEC, IEEE y muchos otros grupos de normalización y organizaciones internacionales que desarrollan protocolos específicos que permiten la transmisión de datos (JPEG, MPEG, ISO, SAC, etc.), los CIR se encuentran en el centro de las fórmulas institucionales que se han venido conformando en estos años y que constituyen el «teatro de la legitimidad $»^{29}$ dentro del que se juega la partida por la «libertad política» en relación a las nuevas tecnologías.

A la vista de esto, Internet está gestionada mediante un sistema de multistakeholder governance: una red descentralizada e internacional de grupos compuestos por miembros de la sociedad civil, empresas privadas, actores estatales, organizaciones internacionales, académicos y muchos otros. La identificación individualizada de los interesados es de por sí complicada ${ }^{30}$, e incluso hay quien diferencia entre distintos tipos de stakeholders en función de su naturaleza o su capacidad de influencia: los "ciudadanos del mundo», los clientes de una determinada compañía de telecomunicaciones, los consorcios de investigación, las empresas, los gobiernos, o las instituciones expresamente dedicadas a la gestión de la red, como pueda ser el IGF, aunque su función sea meramente consultiva. El propio mapa de las instituciones implicadas es complejo, orientándose a su estudio parte de los trabajos actuales que se dedican a esta forma de governance ${ }^{31}$. De hecho, estamos asistiendo a un proceso de creciente institucionalización en este ámbito, lo que demuestra que la gestión de la red se está convirtiendo en una auténtica policy area individualizada en la que se puede encontrar a los grandes actores políticos.

Sin embargo, ni la ciencia ni la teoría política han considerado todavía que este sea un ámbito de interés. Habituados, de modo reflejo, a buscar procedimientos decisionales formalizados, en especial en el marco estatal, muchos no han visto lo que ha estado sucediendo. Tiene razón Helen Margetts, directora del Oxford Internet Institute, cuando escribe que, si bien Internet puede ser «un eventual estímulo para muchos ismos de la teoría política normativa», la mayoría de los estudios sobre el tema - y en particular los más antiguos sobre el impacto de la introducción de los ordenadores y las telecomunicaciones informatizadas en la política y la democracia- han sido simplemente «guetizados por la ciencia política mainstream» ${ }^{32}$.

29 Wagner (2013), también Wagner (2016).

30 Manuel-Navarrete y Modvar (2007).

31 Se pueden ver, entre otros, Mueller (2002), (2010); Paré (2003); Malcolm (2008); Mayer-Schönberger y Ziewitz (2006); Post (2009); Weber (2009); De Nardis (2011).

32 Margetts (2013: 421-422). También Mindus (2014), donde se desarrolla la tesis del atraso de la ciencia política en comparación tanto con la ética de la información como con respecto a la informática jurídica. 
Como ejemplo, se puede observar cómo en la literatura sobre la governance global y sus movimientos dentro de la sociedad civil, Internet no se concibe como un objeto de estudio, sino como un medio que sirve para formular y distribuir el mensaje político. A diferencia de la governance y del multistakeholderism que están de moda en temas como el medio ambiente, los derechos humanos, el mercado, la deuda pública y muchos otros sectores de la política contemporánea, la gestión de la red no se plantea como un área de la política, como una realidad o un ámbito en el que se están produciendo luchas políticas. «Esta circunstancia es extraña porque existen movimientos políticos. Alrededor de temas como la propiedad intelectual (commons) y el acceso al conocimiento (A2K) se articulan lo que se pueden calificar como movimientos sociales transnacionales» ${ }^{33}$.

Internet, que actualmente se ha convertido en la plataforma de comunicación más importante del mundo - como resultado del fenómeno de la convergencia digital, que ha unificado sustancialmente las plataformas de medios, haciendo que usemos la red para llamar, ver vídeos, escuchar música, comprar productos y, en general, relacionarnos con los demás-, constituye ya un $o b$ jeto político. Como la política comercial o los asuntos exteriores, la red es en sí misma un ámbito político, y no únicamente un modo de hacer política como insinúa gran parte de la literatura sobre democracia digital. "Exactamente como la política comercial o la medioambiental, la globalización de las comunicaciones y de la información influye significativamente en los individuos. Pero sus efectos más visibles se confinan a un sector muy concreto de las políticas públicas y, por tanto, a áreas institucionales específicas» ${ }^{34}-\mathrm{y}$ son justamente estas áreas las que hoy están cambiando.

\section{MULTISTAKEHOLDERISM Y CENTROS DE PODER}

Es probable que focalizar la atención sobre los ámbitos institucionales formalizados, como la mayor parte de las investigaciones sobre la gestión de la red ha hecho en los últimos veinte años, no sea lo más útil si queremos comprender cuáles son los centros del poder. Del mismo modo, preguntarse cuáles son los actores, los portadores de intereses involucrados en las instituciones formales, quizá no sea la mejor estrategia para comprender los actuales retos a los que se enfrenta la libertad política. No porque las instituciones formales no sean legítimas, sino porque no necesariamente gozan del poder de, precondi-

33 Mueller (2010: 12).

34 Wagner (2013: 12). 
ción del poder sobre. La historia de las instituciones responsables de la gestión de la red parece dar indicios en este sentido.

En esta historia, como es bien sabido — desde Arpanet a Internet- los Estados Unidos han desempeñado un papel clave, si bien, en cierto modo, en un doble sentido. Por un lado, con el fin de crear el marco que permitiera justificar el statu quo, han defendido la imposibilidad técnica de regular o uniformizar la red: como afirmó de manera muy gráfica Bill Clinton, para un Estado regular Internet es como «clavar gelatina en la pared». Pero por otro lado, Estados Unidos ha intentado sistemáticamente asumir el control sobre partes clave de la infraestructura de la red: en otras palabras, controlar al menos aquello que resulta controlable: esencialmente los CIR y, en especial, el root system. El modo de hacerlo era a través de la ICANN. La Internet Assigned Numbers Authority se ocupa, inter alios, de la tarea de asignar los nombres de dominio a través de la delegación a los registrars (como VeriSign), repartir las direcciones IP, y controlar el root server system y el root zone file.

Desde el nacimiento de la red hasta su muerte en 1998, el pionero de Internet Jon Postel se ocupó de la asignación de las direcciones y los protocolos, dada su condición de director de la división de redes informáticas Information Sciences Institute (ISI) de la University of Southern California, subcontratada por el Ministerio de Defensa norteamericano. Más tarde, esta tarea fue encomendada a la Internet Assigned Numbers Authority (IANA); después, cuando esta institución asumió la gestion de los root server en todo el domain name system, se transformó en la organización conocida como ICANN. $\mathrm{Si}$ se analiza despacio, todo lo acontecido no es más que el resultado de una disputa entre expertos técnicos y el Estado americano.

Tal y como se narra en detalle por Mueller en Ruling the Root (2002), el conflicto que enfrentaba a la "comunidad de Internet», in primis representada en la figura de Postel, con los consejeros de Clinton versaba precisamente sobre el control de esta infraestructura. Finalmente la Administración, amenazando con demandar a Postel, impuso su criterio y, en una semana, este otorgó nuevamente al Estado el pleno control sobre los servidores: «desde entonces, el root file y la teórica autoridad sobre el root quedaron, sin excepciones, en manos de los Estados Unidos» ${ }^{35}$. Sin embargo, a la vista del desarrollo del e-commerce y el riesgo, percibido por las empresas, de que el control estatal pudiera perjudicarlo, se prefirió instituir una autoridad no estatal. Desde entonces, se viene desarrollando un debate sobre la función y la legitimidad de la ICANN, organización no gubernamental sin ánimo de lucro sometida al derecho privado californiano, que ha asumido las funciones IANA. El debate

35 Goldsmith y Wu (2006: 46). 
se concentra especialmente en estos aspectos de la red institucional y, sobre todo, en lo relativo al control por parte de la ICANN del domain name system $\left(\mathrm{DNS}^{36}\right)$, adquiriendo un nuevo impulso con la introducción en 2009 del Governmental Advisory Council dentro de su estructura.

El multistakeholderism que caracteriza a la ICANN se plantea como una respuesta a los riesgos que implica el control de estos CIR por parte del Estado americano. Como consecuencia, la ICANN incluye a actores interesados de otros países y de diversos tipos de organizaciones; se trata de una forma de ordenación interna que viene a identificarse con la etiqueta de multistakeholderism. Desde el principio se vio que la posibilidad de participar reconocida a los distintos grupos no suponía nada ni en relación a sus posibles competencias ni al peso que cada uno pudiera tener en los procedimientos de toma de decisiones. No es momento ahora de exponer la evidente diferencia que existe entre tener voz y tener peso decisional, entre simplemente participar y decidir, una cuestión, por otra parte, ampliamente desarrollada por los estudios críticos sobre la governance, floreciente ámbito de la literatura contemporánea sobre política normativa ${ }^{37}$. En nombre de los principios de inclusión, transparencia y proximidad, en muchos procesos de governance se considera que las decisiones públicas deben ser negociadas entre el mayor número de actores diferentes posible, individuos o grupos, incluso si eso supone relegar a los organismos públicos al papel de simples mediadores. En el caso del multistakeholderism propio de la red, la pretensión era involucrar a distintos grupos en los procesos decisionales, no solo como agentes consultivos. Son muchos los

36 El domain name system, que traduce los nombres de dominio alfanuméricos y sus direcciones IP, es un enorme database management system jerárquico, distribuido globalmente en numerosos servidores. Los root name servers contienen un código maestro conocido como root zone file que gestiona las direcciones IP y los nombres asociados del servidor DNS para todos los TLDs y ccTLDs (dominios top-level y los códigos de países asociados como .com o .it): esto último actualmente está incluido en las funciones de la IANA. Por razones técnicas, existen 13 root servers, que, sin embargo, son gestionados por 12 organizaciones entre las que se encuentra la NASA, el ejército americano y varias compañías privadas, de las que dos son propiedad de VeriSign. La gestión del root zone file la realiza la ICANN, que es quien puede ponerlo al día; la actualización debe ser autorizada por el Ministerio de Comercio americano y el gestor del DNS root server (Verisign) será el encargado de incluir los cambios necesarios y distribuir la actualización a los otros DNS root servers.

37 En italiano se puede consultar Ferrarese (2010). Para comprender la concepción privatista de la representación a partir de intereses y de los procesos de negociación típicos de la governance en relación a la concepción política de la representación, véase Cuono (2013). 
que han apuntado que ya la propia idea ponía en crisis muchas de las concepciones tradicionales sobre la representatividad, la legitimidad y la responsabi$\operatorname{lidad}^{38}$. Pero, sobre todo, la novedad venía dada por el nuevo papel que se asignaba a los «expertos» que, en este caso, eran también los «padres fundadores de la red» en torno a los que se desarrollaba un auténtico y genuino culto a la personalidad ${ }^{39}$. Partiendo de la premisa de que «los políticos no disponen de conocimientos o experiencia sobre el funcionamiento técnico de las redes, se hace necesario que los expertos técnicos mantengan el control sobre una parte del proceso de gestión. Expertos que en un primer momento eran escuchados por las comisiones parlamentarias o las estructuras burocráticas análogas, ahora se sientan en estas mismas comisiones desempeñando un papel activo en el desarrollo de las políticas» ${ }^{40}$.

Así, nos encontraríamos con un sistema que reproduce los vicios más criticables de la expert rule, de la tecnocracia dentro de la democracia, si no fuera por el hecho de que el modelo de multistakeholder presente en la creación del ICANN siempre ha estado bajo la larga mano, apenas oculta, de la hegemonía jerárquica de los Estados Unidos. A este respecto, es curioso cómo en la modernidad política nunca se emplearon «expertos» de modo tan generalizado como se hace en la época de la «muerte del conocimiento» y de la posibilidad indiscriminada de opinar. Mientras que existe una amplia literatura sobre el abuso del saber técnico como forma de tecnocracia en otros ámbitos de la política, como por ejemplo la política financiera y la política migratoria, en relación a la política de las TIC lo más importante parece ser denunciar la influencia de los Estados Unidos o defender el modelo del multistakeholderism de cambios o ataques antes que revelar su carácter dual y la ambigüedad de esta forma de recurso al conocimiento técnico. Esta manera de enfocar el debate, prevalente en los años noventa y al principio de este siglo, sorprende más todavía a la vista de que, al propio tiempo y en relación al mismo ámbito político, se planteaban acusaciones de techdeterminism: ¡cómo es que nunca el experto en redes - ya sea consciente de que la técnica incorpora valores y, por tanto, no es neutral en relación a las distintas formas de ver el mundo, o bien sabedor de que aquella siempre parte de una hipótesis concreta sobre el comportamiento humano- parece preocupado por el hecho de llevar con él, junto con el propio bagaje de conocimientos técnicos, su particular visión del mundo?

\footnotetext{
38 Bendiek y Wagner (2012).

39 Leiner et al. (2011).

40 Wagner (2013: 181).
} 
En cualquier caso, considerando la atención prestada al papel de los Estados Unidos, no es sorprendente que el antiamericanismo entre en el debate reivindicando espacios decisionales alternativos para la gestión de la red. Sin embargo, el multistakeholderism también cumple su función en este contexto: "como organismo privado cuyo fin es la regulación eficaz de la gestión de la red, el ICANN también sirve para deslegitimar la creación de estructuras institucionales paralelas [...]: ya existe un espacio donde los usuarios de la red pueden expresas sus preocupaciones [...]. Esta es la realidad efectiva de la multistakeholder governance: implicar directamente a las ONG, los expertos y las empresas en los procesos de governance es la manera de impedir un control multilateral» por parte de los Estados ${ }^{41}$.

Como consecuencia de esto, aparecen fuerzas contrarias a esta forma de legitimidad prevalente establecida a partir del ICANN. Muchos históricos de la red recuerdan cómo el IGF fue, en muchos sentidos, la respuesta de la ONU a la creación del ICANN ${ }^{42}$. El dictador tunecino Ben Ali consiguió convencer a la $\mathrm{ONU}$ de la necesidad de organizar una conferencia internacional en Túnez sobre el control de la red. Aunque al hacer esto los objetivos del dictador, que no por casualidad fue apodado Ben à vie durante las protestas que le obligaron a abandonar el poder, bien podían ser otros, muy distintos a la conformación de una red «libre», lo que consiguió de hecho fue reabrir la discusión sobre la gestión de la red.

Desde 2003 la governance de Internet se ha discutido en la Cumbre Mundial sobre la Sociedad de la Información (WSIS). La primera reunión se produjo en Ginebra en 2003, bajo presión de los Estados Unidos y otros países aliados, y la segunda en Túnez en 2005. Se trató de una serie de conferencias diplomáticas multilaterales en las que estaban presentes representantes de distintos estados para afrontar aquello que consideraban que constituía el problema de la gestión de la red; en otras palabras, nada menos que los principios, normas, reglas y procedimientos compartidos que conforman la evolución y ordenan el uso de Internet. Hay quien habla de esto como «la batalla por el alma de la red ${ }^{43}$. Lo que estaba en juego era básicamente el dominio americano sobre los CIR, pero también el papel de los Estados en la gestión de la red. Este último punto aparecía en la agenda de Túnez así: «Permitir a los gobiernos, en condiciones de igualdad, desempeñar sus roles y responsabilidades en relación a las políticas públicas internacionales propias de Internet», como expresa la Declaración oficial.

41 Wagner (2013: 179-182).

42 Masters (2014).

43 Ellio Noss, citado por Mueller (2010: 10). 
Justo tras la WSIS de Túnez, en 2006 se creó el IGF, que constituye un grupo internacional de gobiernos y organismos no gubernamentales. Este, llamado a veces «parlamento de Internet», pero autodefinido como «un laboratorio o espacio neutral para el diálogo» ${ }^{44}$, representa un lugar de encuentro para discutir y profundizar sobre los asuntos de mayor actualidad. Pero el IGF no tiene capacidad decisional: si bien algunos lo consideran una «sede de poder» indirecta, en la medida en que puede influir en las prácticas de governance a través de redes informales o procesos de socialización, de la conformación de comunidades y de las relaciones de micro-poder ${ }^{45}$, lo cierto es que carece de capacidad coercitiva alguna. De ahí que haya quien considera que dirigir la atención hacia el IGF, debatir sobre su legitimidad, es un enfoque en buena medida equivocado, ya que las verdaderas sedes del poder son otras ${ }^{46}$. «La principal función del IGF es mantener el statu quo institucional, impidiendo a otras instituciones tomar parte en la gestión de la red ${ }^{47}$. En diciembre de 2010 la ONU decidió proseguir con la experiencia del IGF por otros cinco años; sin embargo, la estrategia no parece haber funcionado.

De hecho, tanto algunos Estados como el propio sistema internacional temen que el lock-in institucional — que se produce cuando un órgano, aunque sea consultivo, reivindica su capacidad para expresarse sobre una materia tan sensible como la gestión de la red- pueda minar el poder de otros entes con capacidad decisional, en particular, la Unión Internacional de Telecomunicaciones (ITU), encargada de organizar la WSIS. Tanto la legitimidad de la WSIS como la del IGF derivan de la agenda de Túnez, pero en realidad no están del todo alineadas, sino que son instituciones concurrentes. A esto se ha unido también la Unesco que, en febrero de 2013, organizó en París un fórum de la WSIS. E incluso los Estados han querido participar: en diciembre de 2011 se formó en La Haya la Freedom Online Coalition, cuya última reunión se produjo en Tallin en abril de 2014. Forman parte de ella Austria, Canadá, la República Checa, Costa Rica, Estonia, Finlandia, Francia, Georgia, Alemania, Ghana, Irlanda, Japón, Kenia, Letonia, las Maldivas, México, Moldavia, Túnez, el Reino Unido, y desempeñan papeles importantes Holanda, Suecia y Estados Unidos. En algunos de los eventos organizados por este foro alternativo de discusión han participado más políticos y se ha recibido mayor cobertura mediática que en los del IGF. En conclusión, desde el 2005 hasta hoy

\footnotetext{
44 http://www.intgovforum.org/cms/aboutigf.

45 Flyverbom (2011), que estudia principalmente WGIG y UNICTTF, a partir de una aproximación STS.

46 DeNardis (2013: 557).

47 Wagner (2013: 185).
} 
en día se ve cómo la escena se va fragmentando cada vez más en lo que respecta al ámbito institucional que se ocupa de la gestión de la red. De hecho, se ha asistido a una proliferación de instituciones muy diferentes. "El resultado ha sido la exclusión del IGF de los debates más importantes sobre la gestión de la red, mientras que los Estados y las organizaciones internacionales se van inventando nuevos puntos de encuentro ${ }^{48}$. No hace falta ser un empedernido realista político para reconocer que el interés por un determinado foro de encuentro depende en parte del poder que se piensa que éste puede tener, y que esta fragmentación institucional es una prueba de los cambios en el paisaje del poder.

Sin embargo, la pervivencia del modelo de multistakeholder governance ha sido posible gracias al papel clave desempeñado por los Estados Unidos. Mientras que este país se resista a los cambios, será complicado variar la ruta, lo cual no impide, sin embargo, que se hagan esfuerzos en la dirección opuesta. De hecho, los detractores del modelo de gestión de la red consiguieron, bajo la batuta del ITU, organizar a finales de 2012 una conferencia internacional de alto nivel en Dubái, la WCIT (World Conference on International Telecommunications). «Algunos puntos de la conferencia WCIT afrontaban auténticas cuestiones de poder sobre la gestión de la red. Por ejemplo, el hecho de otorgar un mandato al ITU para regular la "cybersecurity" va directo al corazón del modelo del multistakeholderism» ${ }^{49}$. Rusia, China, Arabia Saudita, Argelia y Sudán aprovecharon la ocasión para declarar que «los Estados miembros tienen el derecho soberano de establecer e implementar políticas públicas, también a nivel internacional, en cuestiones relacionadas con la Internet governance ${ }^{50}$. La propuesta también aspiraba a devolver a los Estados miembros la responsabilidad sobre gran parte de las funciones de la ICANN ${ }^{51}$. A este paso, la WCIT acabará por obligar a los Estados Unidos a defender abiertamente el modelo del multistakeholderism.

Es preciso también señalar que, en los últimos años, se ha visto cómo las instituciones típicas de este modelo de governance carecen de poder real para

48 Ibid.

49 Wagner (2013: 191).

50 ITU-SG WTPF13IEG3, Documento WTPF-IEG/3/35: Contribution from the Russian Federation. Opinion (7): The role of Member States in the Internet Governance Multistakeholder model to facilitate its further development, accesible en la dirección: http://www.itu.int/md/S13-WTPF13IEG3-C-0021/en

51 Ibid.: «Los Estados miembros tienen los mismos derechos sobre la gestión de Internet, entre los que están los derechos al reparto, a la asignación y a la reutilización de códigos, direcciones, dominios y recursos, así como al desarrollo de infraestructuras de base de la red». 
incidir en la práctica política. Aunque el modelo aún sea defendido por individuos y grupos que operan dentro de él ${ }^{52}$, se trata de una fórmula cada vez más vacía, en el sentido de que la capacidad para influir en la gestión de la red por parte de este conglomerado de fuerzas institucionales está muy limitada. De hecho, hoy la red se gestiona a través de relaciones y acuerdos normalmente de tipo informal, esencialmente entre actores privados. Por mucho que los actores públicos, ya sean de nivel nacional o internacional, intervengan en la determinación de las pautas políticas dentro de este ámbito, su capacidad para influir, condicionar o determinar el comportamiento del resto de jugadores y de los propios usuarios está muy limitada. Estos actores carecen, en otros términos, de poder sobre la política de la red. Pero ¿por qué sucede esto?

Un aspecto que parece distinguir a este nuevo sector de la política de muchos otros (inmigración, mercado de trabajo, política exterior, etc.) es que, en el sector comunicativo-informativo, «la confianza en el Estado nación como principal institución gubernativa es exactamente lo que se pone en cuestión ${ }^{53}$. Bajo el slogan code is fast, law is slow, se oculta una de las principales paradojas de este ámbito político: su esquizofrénica relación con el Estado.

Por un lado, el Estado sigue siendo la enforcement agency por excelencia, aunque la red globalizada sea un animal elusivo que ningún Estado está capacitado para controlar plenamente. Aquellos que pensaban, haciendo gala de un entusiasmo indisimulado, que los Estados desaparecerían en la infoesfera sencillamente estaban equivocados. Los Estados plantean pretensiones sobre nombres geográficos o sobre representaciones lingüísticas en la infoesfera, desarrollan su propia capacidad para ejercer vigilancia, hacen planes para militarizar el ciberespacio y colonizar aquellos elementos considerados esenciales y pretenden ser los árbitros de la censura en la red. Todas estas son formas mediante las que se manifiesta el más clásico poder sobre los netizens. Por otro lado, el Estado parece más que otra cosa un tigre de papel: quiere, pero no puede. De hecho, carece de poder de, en el sentido de los medios técnicos necesarios para gobernar la política de la red. Muchas veces los Estados, cuando intentan regular los algoritmos de las compañías privadas y no solo su comportamiento, simplemente fracasan. Han perdido la capacidad de intervención (poder de). E incluso, cuando se esfuerzan en mantenerla, se muestran incapaces de ello. Por ello, también se equivocaban aquellos que teorizaban, como aplicados deterministas tecnológicos y empedernidos realistas políticos, que estábamos condenados a vivir en closed source societies con una red de fronteras rígidas bajo la vigilancia estatal. El Estado —se podría decir aplican-

52 Franklin (2013).

53 Mueller (2010: 255). 
do la tesis de Saskia Sassen en el debate de los años noventa sobre el declive de la soberanía- «decae creciendo».

Pero mientras que las expectativas depositadas sobre el Estado aumentan, este ve reducida su capacidad para emplear aquellos medios que necesita para producir el efecto que desearía. En este sentido, los actores públicos no son muy libres, lo que significa, en el caso de los Estados democráticos de derecho, que tampoco nosotros lo somos, en la medida en que los ciudadanos son el componente básico del Estado. La falta de libertad de un Estado, en este contexto, no depende esencialmente del hecho de que otro ejerza poder sobre él, sino de la falta de poder de. Esto no sucede, como alguno podría estar tentado de creer, como consecuencia del poder que ejercen los Estados Unidos sobre algunos CIR, el DNS in primis, sino como resultado de que el propio poder en la red ha mutado a causa de los cambios en el comportamiento de los usuarios, condicionado a su vez por lo que ofrecen los provider y data brokers.

El multistakeholderism, como modelo de gestión que actualmente está siendo cuestionado por una pluralidad de actores, ha consentido que algunos jugadores adopten posiciones dominantes. Empleando las palabras de un senior official en Internet governance con mucha experiencia:

El multistakeholderism es algo fatalmente defectuoso, al menos en el contexto de la Internet governance, a causa de (a) su vaguedad, y (b) su (ab)uso por parte de algunos poderes preestablecidos como tapadera para sus propios intereses estratégicos; algo directamente relacionado con el punto (a) en la medida en que la tiranía de la falta de estructura permite a los poderes preestablecidos guiar las decisiones a través de la mano invisible del multistakeholderism ${ }^{54}$.

En realidad, esta es una característica ya denunciada en relación a muchos procedimientos de governance no tradicional desarrollados en otros ámbitos políticos, por lo que, como tal, no debería sorprender. Así que convendría centrar la atención en los eventos más recientes para entender qué ha pasado.

Desde 1997, es decir, desde el momento en que la gestión de estos CIR se transfirió del ejército de los Estados Unidos a manos privadas, el control último sobre la root zone se confió a la National Telecommunications and Information Administration (NTIA), que opera bajo la dirección del Departamento de Comercio americano. Ya en 1998 la propia agencia anunció que llevaría a cabo «una transición que permitiera al sector privado asumir el lide-

54 Véase Wagner (2013: 173). 
razgo en la gestión del DNS», aunque nunca lo hizo. Sin embargo, con el modelo del multistakeholderism entrado en crisis, en marzo de 2014, tras el $49^{\circ}$ encuentro de la ICANN en Singapur, la NTIA anunció que la autoridad sobre la root zone será cedida a la "comunidad global de interesados». El contrato de la ICANN para desempeñar las funciones de la IANA acababa el 30 de septiembre de 2015. El teatro de la legitimidad de este nuevo ámbito de la política está, por tanto, en plena transformación.

Hay quien ha sostenido que este movimiento por parte de la NTIA debe ser interpretado como una respuesta al escándalo datagate en el que se ha visto involucrado la NSA (United States National Security Agency) ${ }^{55}$. Las revelaciones de Snowden han tenido sin duda repercusiones importantes en las relaciones internacionales, sirviendo en buena medida como aviso para que muchos Estados intenten prevenir el espionaje de datos a través de las grandes cadenas de comunicación. La entonces presidenta brasileña Dilma Rousseff se reunió en febrero de 2014 con François Hollande para comunicarle su intención de tender un cable submarino entre Brasil y Europa con el fin de evitar que el tráfico de Internet de su país pasara a través de los Estados Unidos. Poco antes, la presidenta alemana Angela Merkel manifestó su voluntad de debatir sobre la creación de una «Internet europea» con el fin de mantener el tráfico de los ciudadanos de la UE dentro de los límites europeos sin pasar por los Estados Unidos por el mismo motivo. Ante estos anuncios, no hay que sorprenderse si algunos alzan la voz alertando del riesgo de una balcanización de la red. De hecho, estos eran los temas del orden del día en el encuentro Netmundial de São Paulo en abril de 2014 y en la reunión del IGF de finales de agosto del mismo año.

No obstante, parecen existir otros motivos, más profundos, que sugieren un cambio de ruta en la gestión de la red. Derivan esencialmente de la manera en la que ha cambiado el modo de usarla.

\section{PODER DE Y PODER SOBRE}

En el lapso de tiempo transcurrido entre la «privatización» de las funciones de la IANA, bajo la dirección del Ministerio de Comercio americano, y el anuncio "histórico» de renuncia a ellas, han sucedido, de hecho, muchas cosas: hemos asistido a la llegada de los big players. Por una parte, las grandes compañías del sector privado; por otra, los Estados. Hay quien sostiene que en los últimos veinte años, dado el creciente poder de algunos jugadores de

55 Para comprender el impacto del datagate y sus repercusiones en el mundo digital, véase Chiusi (2014). 
condicionar el comportamiento de los demás, hemos pasado a vivir bajo la sombra de un modelo de gestión privada de la red ${ }^{56}$. Por otro lado, la propia red ha cambiado, como resultado de los cambios en el comportamiento de los usuarios.

Es digno de mención que, en efecto, la variación en la forma de usar la red por parte de los sujetos ha motivado una de las mayores modificaciones a nivel de su control. De hecho, el sistema del root y de los nombres de dominio tiene mucha menos importancia hoy que hace veinte años. Los usuarios han encontrado maneras de circunnavegar los puntos de entrada en el sistema gracias en buena medida al desarrollo de instrumentos por parte de las grandes empresas de Silicon Valley. En concreto, los motores de búsqueda como Google se han convertido en sustitutos de facto del DNS dado que - al tiempo que aumenta el tiempo que se pasa online en buscadores o redes sociales-el buscador de esta compañía, con su barra de acceso rápido, permite a los usuarios llegar fácilmente a sitios web cuya dirección exacta no recordaban. El motor de búsqueda se convierte así en el principal punto de ingreso a mercados donde se mantiene un monopolio sobre las búsquedas en la red; un comportamiento no muy diferente también se observa en el empleo de Facebook en algunas partes del mundo. Para el usuario es más sencillo utilizar estos servicios que memorizar el listín telefónico de direcciones - lo cual conduce al progresivo olvido del mismo-. "Al tiempo que ha ido cambiando el comportamiento de los usuarios, la ICANN se ha ido convirtiendo en algo irrelevante como institución. Así, hoy es, sobre todo, una institución simbólica que muestra el palco más visible del "teatro de la legitimidad", pero con poco acceso a la política que se desarrolla tras las bambalinas»" ${ }^{57}$.

Por tanto, recapitulando, en el momento en el que la institución era aún relevante porque ofrecía la principal puerta de acceso al sistema se permitió que aquellos que pudieran tener algún interés, en particular las empresas tecnológicas, se implicaran de modo directo en sus procesos decisionales. En otras palabras, se consiguió que un espacio en el que se debían tomar decisiones de alcance erga omnes para quien quisiera usar el medio no fuera ocupado por instituciones estatales, comités parlamentarios, entes burocráticos o por el control de los tribunales. Lo más probable es que tal decisión estuviera motivada por el deseo de dejar a las empresas de Silicon Valley desarrollar su tecnología. Lo que no está claro es si se hubiera hecho lo mismo ante la voluntad de una empresa extranjera de establecer un monopolio sobre los motores de búsqueda. Por el contrario, hoy se reconoce sin problemas el «importante

56 Wagner (2013: 65).

57 Wagner (2013: 182). 
papel que reviste la iniciativa privada en la determinación de la política online [...]. Representantes de las compañías tecnológicas participan activamente en las instituciones encargadas de determinar los estándares», por lo que se habla de un "paso en la dirección opuesta a la marcada por los valores tradicionales de Internet. Las formas de gestión de la red pueden ser intrínsecamente técnicas para ganar ventaja sobre la competencia económica ${ }^{58}$. Tras la restricción autoimpuesta por el Estado americano sobre la gestión de la red, la ICANN consintió que se desarrollase una nueva generación de formas de poder en la red, vaciándose finalmente de competencias. Citando a Shakespeare, se puede decir que habiendo cumplido su cometido, el Moro puede irse.

Con estos cambios, sin embargo, ha aumentado sustancialmente la capacidad regulatoria de la red, incrementándose notablemente las posibilidades de varios operadores para incidir significativamente sobre el comportamiento de los individuos. La gran novedad de los últimos veinte años es la capacidad de los ISP para filtrar contenidos automáticamente. Se puede, con razón, hablar de un incremento de la regulación en los últimos veinte años como correlato de la transición desde los ISP a los OSP (Online Service Providers, como las plataformas de Google, Microsoft, Facebook, Yahoo! etc.). La capacidad para influir sobre los comportamientos implica, por ejemplo, que, a diferencia de lo que pasaba antes, ahora un servicio de email puede filtrar texto, imágenes, etc. ${ }^{59}$; ahora se analizan los archivos almacenados en la nube para filtrar material ilícito mientras que antes no se filtraba el contenido del disco duro del ordenador doméstico; por defecto se filtra el material en las LAN de compañías privadas y públicas; Apple, que goza de una posición de preeminencia con sus iPhone, puede purgar las aplicaciones de su propia store con amplia discrecionalidad, y así sucesivamente. A nivel de contenidos, «un pequeño número de proveedores actúa como puerta de entrada para la mayor parte de los usuarios. Un puñado de empresas — Baidu, Google, Sina, Facebook, Twitter y otros- dominan las búsquedas, los social media, y el social networking en red $»^{60}$.

Las normas elaboradas por plataformas privadas para ordenar la actividad en sus sitios web han asumido el papel de pautas sobre el comportamiento de los usuarios, orientando y condicionando su actuación. A veces estas reglas están en consonancia con las leyes nacionales de los usuarios, pero lo normal es que no sea así en absoluto. Cuando Bing filtra los resultados para los usuarios en lengua árabe, o YouTube suspende la cuenta de un usuario por

58 De Nardis (2013: 571-572).

59 McIntyre (2012).

60 Faris y Heacock (2013: 2). 
la presencia de material violento, estos actores privados desempeñan de hecho la función de reguladores, y muchas de sus decisiones se adoptan fuera del control público formal. No se trata de «leyes» por diversas razones. Ya sea porque carecen de la abstracción y la generalidad de estas - la mayor parte de las decisiones operativas en los network gate keepers o content regulatory agents ${ }^{61}$ son adoptadas por individuos y normalmente asumidas por las organizaciones con el fin de filtrar contenidos, cosa que tiende a realizarse de manera discrecional, cuando no de forma directamente arbitraria, y siempre sobre la base de criterios ad hoc - ya sea porque no se trata de normas, sino de impedimentos técnicos a la realización de un determinado comportamiento (por ejemplo, a través del parental control filtering), de modo no muy diferente a lo que sucedía en el caso de las antiguas DRM. Si bien algunos esperan que con la adopción del http 451 en diciembre de 2015 sea posible tener más capacidad para distinguir entre los errores técnicos y los errores de carga de una página debidos a razones legales, y por tanto recoger estadísticas más fiables sobre la censura en la red, otros subrayan cómo algunos sitios podrán verse obligados a emplear el http 404 (file not found) a pesar de que exista la posibilidad técnica de beneficiarse del estándar $451^{62}$.

Hace ya algún tiempo, el Monitor 2013 del Berkman center advertía que

[...] las grandes empresas de las redes sociales operan de facto como árbitros en la fijación del discurso aceptable en la red. Apenas estamos empezando a comprender cuánta discrecionalidad poseen las grandes empresas para modular la eficacia de la vigilancia estatal, tanto si cumplen rápido con las solicitudes que les hacen los Estados para obtener información como si las rechazan. Aunque no sea una consecuencia del diseño, un número importante de intereses públicos dependen de la backbone infrastructure de naturaleza privada, donde las decisiones se toman en base a criterios de mercado.

\section{Robert Faris y Rebekah Heacock constataban así que}

[...] las empresas forman el núcleo funcional de Internet. El sector privado posee una vasta parte de la infraestructura física, produce el hardware y el software que se usan en la red, desarrolla aplicaciones y servicios innovadores,

61 Content regulatory agents son por ejemplo el Facebook Hate and Harassement Team o bien la Freiwillige Selbstkontrolle Medienanbieter (FSM). Esta última es una ONG alemana, financiada por el Estado, con sede en Mainz. Véase http://www.fsm.de/en. http://www.wired.co.uk/news/archive/2015-12/21/error-451-internet-censorshipalert. 
opera como gateway para el acceso privado y empresarial a Internet y aloja la parte más significativa de los contenidos y la información [...]. Como intermediarios entre los gobiernos y los ciudadanos y entre ciudadano y ciudadano, las empresas tecnológicas vienen a desarrollar diversas funciones, en ocasiones incompatibles entre sí: colaboran con las fuerzas del orden, protegen a los usuarios de violaciones de la seguridad y de la excesiva indiscreción de los gobiernos o de terceros, y determinan normas de comportamiento en sus plataformas (de hecho, legislando) [...]. Este nuevo poder de los intermediarios sustituye al que tradicionalmente residía en la judicatura ${ }^{63}$.

Por ahora la red parece ser un espacio libre para una serie de actores muy distintos. Al menos es libre para algunos grandes poderes económicos y mediáticos; y parece libre en esencia respecto de los vínculos que impone el Estado democrático de derecho. Si bien en Silicon Valley las siglas cambian rápidamente, en la política de Internet podemos esperar que el poder no público, o private ordering, con independencia de cuáles sean las empresas, continúe desempeñando un papel esencial. Un primer e importantísimo motivo para ello es que dispone del poder de: de los medios y, por ahora, también de las facultades. Por su parte, el poder público está desarrollando actualmente, a distintos niveles, políticas de reclaim sobre un ámbito que se ha convertido en demasiado importante como para ser abandonado en manos de otros. Obviamente los Estados disponen de la amenaza, no siempre muy eficaz, que supone la capacidad regulatoria, pudiendo convertir en ilícitas conductas que antes pertenecían a la esfera de la libertad de algunos agentes. Las consecuencias de estos esfuerzos normativos por parte de los poderes públicos son, por ahora, más bien decepcionantes (siempre que no se añadan factores generadores de miedo - recordemos que muchos Estados no son ni democráticos ni liberales)_. No existe un sistema regulatorio coherente y operativo que permita regular la actividad en la red. Una de las mayores batallas políticas gira alrededor de la cuestión de si el Estado debe tener un papel activo al respecto. Y, bien interpretada, no se trata de una lucha entre distintas opciones políticas por conseguir el poder, sino de un debate para fijar cuánto espacio dejar a qué poderes sociales. Para orientarse en esta batalla es necesario entender que no es cierto que aquellos ámbitos en los que no interviene el poder político sean ámbitos donde no existe la subordinación. Son espacios libres, sí, pero solo de un tipo de intromisiones muy concretas, las que lleva a cabo el Estado; no son espacios libres de toda clase de poder. La casa, el oikos, se considera tradicionalmente como algo ajeno al reino de la política, pero las formas más clásicas

63 Faris y Heacock (2013: 4). 
del poder son justamente las que se dan en ella: el poder del marido, del padre o del patrón.

La controvertida sentencia sobre el derecho al olvido del TJUE —el fallo Costeja González de 8 de mayo de 2014- trae a colación muchas de las cuestiones planteadas desde la posibilidad de imponer restricciones a los proveedores de Internet que, de forma similar a los embajadores, en las sociedades liberales no sufren castigo. En última instancia, se trata de la relación entre dos tipos de poderes sociales, donde los embajadores representan al poder económico y mediático y quienes quieren castigarlos al poder político. Pero ahora debemos comenzar a preguntarnos: ¿por qué se acepta que el embajador pueda ser castigado en unos casos pero no en otros? ¿Por qué un ISP puede ser reclutado para proteger los derechos de autor y otros derechos económicos, pero no los derechos fundamentales de otra clase? ¿Estamos seguros de que borrar la información es el modo de impartir justicia ante la violación de un derecho? ¿No es esto acaso una primitiva confusión entre venganza y justicia? Si el Estado, entendido como la colectividad de los ciudadanos, quiere seguir siendo - aunque sea en el mar siempre cambiante de la dialéctica entre la libertad de los unos y el poder de los otros- quien determine la propia forma de vida, debe actualizarse drásticamente para asumir mayor poder de -medios, capacidad y facultades para estar efectivamente en condiciones de lograr los resultados que desea. De otro modo, pretender ejercer un poder sobre es flatus vocis. $\mathrm{Y}$ "como ciudadanos, debemos estar atentos para no convertirnos en nuestros peores enemigos o jugar inconscientemente las cartas de la mano invisible. Dado que los individuos son la última rueda del carro de la red $-\mathrm{y}$ lo son sin duda - debemos permanecer unidos ${ }^{64}$. Se debe evitar, de hecho, que cualquier poder, independientemente de que se base en instrumentos de manipulación de la conciencia, en la obstaculización del disfrute de bienes esenciales para la supervivencia, o en el recurso a la coerción violenta sobre el cuerpo, nos reduzca a la condición de siervos, privados por tanto de la libertad política.

\section{Bibliografía}

Bendiek, A. y Wagner, B. (2012). Internationale Politik, 67 (6), 85-92.

Benkler, Y. (2000). From Consumers to Users: Shifting the Deeper Structures of Regulation Towards Sustainable Commons and User Access. Federal Communications Law Journal, 52, 561-579.

Bobbio, N. (2009). Teoria generale della politica. Torino: Einaudi.

64 Powles (2014). 
Burkart, P. (2014). Pirate Politics: The New Information Policy Contests. Cambridge (MA): The MIT Press.

Cerf, V. (2004). On the evolution of Internet technologies. Proceedings of the IEEE, 92 (9), 1360-1370. Disponible en: http://dx.doi.org/10.1109/JPROC.2004.832974.

Chiusi, F. (2014). Edward Snowden: A Year Later. Disponible en: http://techpresident.com/ news/25101/edward-snowden-year-later.

Cuono, C. (2013). La rappresentanza democratica alla prova della governabilità e governance. Fenomenologia e società, 34 (1), 53-66.

DeNardis, L. (ed.) (2011). Opening Standards: The Global Politics of Interoperability. Cambridge (MA): The MIT Press.

- (2013). The Emerging Field of Internet Governance. En W. H. Dutton (ed.). The Oxford Handbook of Internet Studies (pp. 555-575). Oxford: Oxford University Press.

Dutton, W. H. (2011). Freedom on the Net 2011. Disponible en: https://freedomhouse.org/ report/freedom-net/freedom-net-2011\#.VIA9FdKG_HQ.

Dutton, W. H. y Peltu, M. (2007). Emerging Internet Governance Mosaic: Connecting the Pieces. Information Polity, 12 (1-2), 63-81.

Faris, R. y Heacock, R. (2013). Internet Monitor 2013: Reflections on the Digital World. Harvard University, Berkman Center for Internet and Society. Disponible en: https://cyber. law.harvard.edu/publications/2013/reflections_on_the_digital_world.

Ferrarese, M. R. (2010). La governance fra politica e diritto. Bologna: il Mulino.

Flyverbom, M. (2011). The Power of Networks: Organizing the Global Politics of the Internet. Cheltenham: Edward Elgar Publishing Limited. Disponible en: http://dx.doi. org/10.4337/9780857936462.

Franklin, M. I. (2013). Digital Dilemmas: Power, Resistance and the Internet. Oxford: Oxford University Press.

Goldsmith, J. L. y Wu, T. (2006). Who Controls the Internet? Illusions of a borderless world. Oxford: Oxford University Press.

Hintz, A. (2007). Deconstructing multistakeholderism: The discourses and realities of global governance at the World Summit on the Information Society (WSIS).

Hintz, A. y Milan, S. (2009). At the margins of Internet governance: grassroots tech groups and communication policy. International Journal of Media and Politics, 5 (1-2), 23-38. Disponible en: http://dx.doi.org/10.1386/macp.5.1-2.23_1.

Hunter, D. (2003). ICANN and the Concept of Democratic Deficit. Loyola of Los Angeles Law Review, 36 (3), 1149-1183. Disponible en: http://dx.doi.org/10.2139/ssrn.400000.

Kallinikos, J. (2011). Governing Through Technology. Information Artefacts and Social Practice. Basingstoke: Palgrave Macmillan. Disponible en: http://dx.doi.org/10.1057/9780230295148.

Leiner, B. M. et al. (2011). Brief History of the Internet. The Internet Society (ISOC). Disponible en: http://www.internetsociety.org/internet/what-internet/history-internet/ brief-history-internet.

MacLean, D. (2005). Governing the Internet as Medium and Message, Model and Metaphor. Governance Forum Position Paper, Oxford Internet Institute.

Malcolm, J. (2008). Appraising the Success of the Internet Governance Forum, Internet Governance Project Paper. Disponible en: http://www.internetgovernance.org/wordpress/wpcontent/uploads/MalcolmIGFReview.pdf. 
Manuel-Navarrete, D. y Modvar, C. (2007). Stakeholder. En M. Bevir (ed.). The Encyclopedia of governance (pp. 918-923). London: Sage.

Margetts, H. (2013). Internet and Democracy. En W. H. Dutton (ed.). The Oxford Handbook of Internet Studies (pp. 421-437). Oxford: Oxford University Press.

Masters, J. (2014). What is Internet governance? Disponible en: http://www.cfr.org/internet-policy/internet-governance/p32843.

Mayer-Schönberger, V. y Ziewitz, M. (2006). Jefferson Rebuffed: The United States and the Future of Internet Governance. John F. Kennedy School of Government Working Paper. Disponible en: http://www.researchgate.net/publication/4895663_Jefferson_Rebuffed-The_United_States_and_the_Future_of_Internet_Governance.

McIntyre, T. J. (2012). Child Abuse images and Cleanfeeds: Assessing Internet blocking systems. En I. Brown (ed.). Research Handbook on Governance of the Internet (pp. 277-307). Cheltenham: Edward Elgar Publishing Limited.

Mindus, P. (2014). What Does E- Add to Democracy?: Designing an Agenda for Democracy Theory in the Information Age. En J. Bishop (ed.). Transforming Politics and Policy in the Digital Age (pp. 200-223). Hershey (PA): IGI Global.

- (2015). Political Liberty and New Technologies. Ragion Pratica, 2015/1, 9-36. Disponible en: https://www.rivisteweb.it/doi/10.1415/79624.

Mueller, M. L. (2002). Ruling the Root: Internet Governance and the Taming of Cyberspace. Cambridge (MA): The MIT Press. Disponible en: http://dx.doi.org/10.7551/mitpress/9780262014595.001.0001.

- (2010). Networks and States: The Global Politics of Internet Governance. Cambridge (MA): The MIT Press.

Nash, V. (2013). Analyzing Freedom of Expression online: Theoretical, empirical, and normative contributions. En W. H. Dutton (ed.). The Oxford Handbook of Internet Studies (pp. 441-463). Oxford: Oxford University Press.

Paré, D. (2003). Internet Governance in Transition: Who is the Master of This Domain? Oxford: Roman and Littlefield Publishers.

Post, D. (2009). In Search of Jefferson's Moose: Notes on the State of Cyberspace. Oxford: Oxford University Press.

Powles, J. (2014). What can we salvage from the "right to be forgotten" ruling. Disponible en: http://www.wired.co.uk/news/archive/2014-05/15/google-vs-spain.

United States Department of Commerce. (2014). NTIA Announces Intent to Transition Key Internet Domain Name Functions, Washington. Disponible en: http://www.ntia.doc. gov/press-release/2014/ntia-announces-intent-transition-key-internet-domain-name-functions.

Wagner, B. (2013). Governing Internet Expression: The International and Transnational Politics of Freedom of Expression. $\mathrm{PhD}$ thesis. Firenze: European University Institute.

- (2016). The Global Default and Algorithmic Regulation: Shifting norms in Internet Technology. En P. Mindus y N. Säfström (eds.). Nordic Journal of Applied Ethics, special issue, forthcoming 2016/1.

Wagner, B. y Mindus, P. (2015). Multistakeholder Governance and Nodal Authority: Understanding Internet Exchange Points. Estudio de casos para el proyecto piloto parte de la Berkman Center's NoC Internet Governance Case Studies Series, en colaboración con Gio- 
vanni Sartor, Uppsala, European University Institute y el Departamento de Filosofía. Disponible en: https://publixphere.net/i/noc/page/IG_Case_Study_Multistakeholder_ Governance_and_Nodal_Authority_Understanding_Internet_Exchange_Points.

Weber, R. H. (2009). Shaping Internet Governance: Regulatory Challenges. Zurich: Springer.

Weinberg, J. (2000). ICANN and the Problem of Legitimacy. Duke Law Journal, 50 (1), $187-$ 260. Disponible en: http://dx.doi.org/10.2307/1373114.

Weiser, P. (2001). Internet Governance, Standard-setting and Self-regulation. Northern Kentucky Law Review, 28 (4), 822-831.

- (2009). The Future of Internet Regulation. Law Legal Studies Research Paper. Boulder: University of Colorado.

Weller, D. y Woodcock, B. (2013). Internet Traffic Exchange: Market Developments and Policy Challenges. OECD Digital Economy Papers, 207, OECD Publishing. Disponible en: http://dx.doi.org/10.1787/5k918gpt130q-en.

Working Group on Internet Governance. (2005). Annual report 2005. Disponible en: http:// www.wgig.org/docs/WGIGREPORT.pdf. 